\title{
Influence of Semicircular Canal Dehiscence on Cochlear Implant Outcome
}

\author{
Jana Matic ${ }^{a, b}$ Sebastian Winklhofer ${ }^{a, c}$ Flurin Pfiffner ${ }^{a, b} \quad$ Christof Roosli ${ }^{a}, b$ \\ Dorothe Veraguth $^{\mathrm{a}, \mathrm{b}} \quad$ Alexander Huber $^{\mathrm{a}, \mathrm{b}} \quad$ Adrian Dalbert $^{\mathrm{a}, \mathrm{b}}$ \\ aUniversity of Zurich, Zurich, Switzerland; b Department of Otorhinolaryngology-Head and Neck Surgery, University \\ Hospital Zurich, Zurich, Switzerland; 'Department of Neuroradiology, University Hospital Zurich, Zurich, Switzerland
}

\section{Keywords}

Semicircular canal dehiscence · Cochlear implantation · Cochlear implant $\cdot$ Hearing preservation $\cdot$ Word perception

\begin{abstract}
Introduction: Semicircular canal dehiscence (SCD) is defined as a defect of the bone overlying the semicircular canal. It has a relatively high prevalence of $3 \%$ in the general population, which makes it likely that a certain number of patients receiving a cochlear implant $(\mathrm{Cl})$ would have it. However, little is known about the influence of SCD on the $\mathrm{Cl}$ outcome. Therefore, the aim of this study was to determine the influence of SCD on $\mathrm{Cl}$ outcome with regard to short- and longterm word perception and hearing preservation. Methods: This study was a retrospective analysis of postoperative word perception ability in the electric-only condition after 6 , 12 , and $\geq 18$ months and of hearing preservation 4 weeks after surgery in $\mathrm{Cl}$ recipients with and without SCD. All patients received a preoperative 1.5- or 3-tesla magnetic resonance imaging. Results: Fifty-five patients were included. Fortyeight patients (87\%) had no SCD, and 7 patients (13\%) had
\end{abstract}

karger@karger.com www.karger.com/aud

Karger"

BOPEN ACCESS
(C) 2020 The Author(s)

Published by S. Karger AG, Basel

This is an Open Access article licensed under the Creative Commons Attribution-NonCommercial-4.0 International License (CC BY-NC) (http://www.karger.com/Services/OpenAccessLicense), applicable to the online version of the article only. Usage and distribution for commercial purposes requires written permission.
SCD. Mean postoperative word perception scores were $66 \%$ in the non-SCD group versus $50 \%$ in the SCD group $(p=0.17)$ after 6 months, 74 versus $64 \%(p=0.28)$ after 12 months, and 77 versus $73 \%(p=0.62)$ after 18 or more months. The mean postoperative hearing loss in patients with functional residual hearing before surgery $(n=34)$ was $22 \mathrm{~dB}$ in the non-SCD group versus $31 \mathrm{~dB}$ in the $\mathrm{SCD}$ group $(p=0.15)$. Conclusions: $\mathrm{Cl}$ outcome is comparable between recipients without and with SCD. Specifically, hearing preservation rate and word perception ability in the electric-only condition seem not affected by SCD. The rate of progress of word perception ability in the first 12 months after cochlear implantation is not influenced by SCD.

(c) 2020 The Author(s) Published by S. Karger AG, Basel

\section{Introduction}

Semicircular canal dehiscence (SCD) is defined as a defect of the bone overlying the semicircular canal. This condition was first described by Minor et al. [1998]. The prevalence of SCD is estimated to be around 3\% in the 

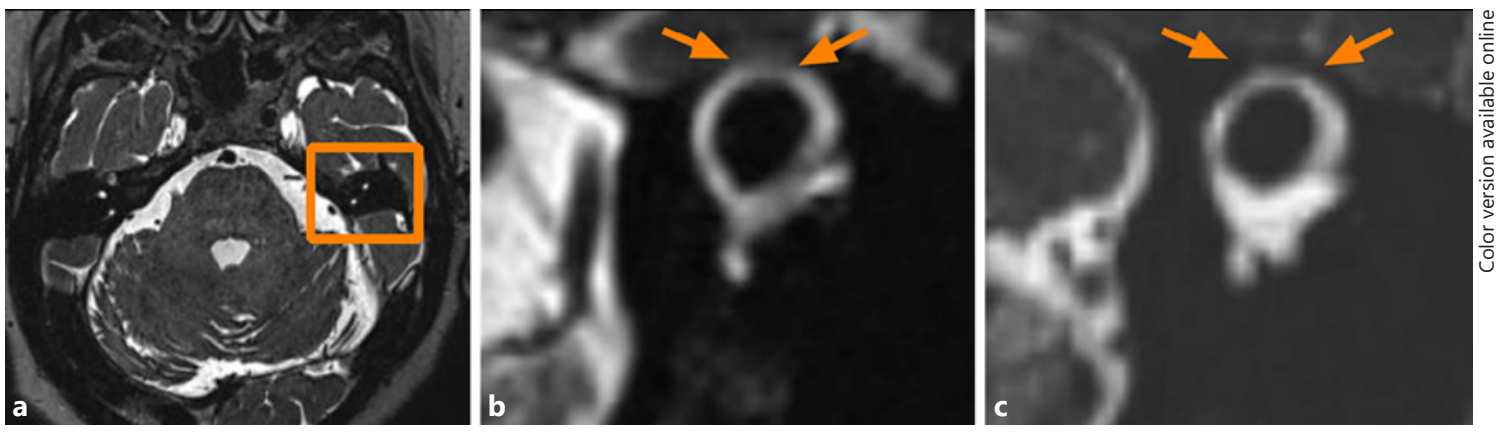

Fig. 1. MRI scan (3-tesla T2-weighted SPACE sequence) of the temporal bone of 2 adults with dehiscence (a, b) and without dehiscence (c) of the left SCC. Axial image reconstruction of the left SCC (a) and magnified image reconstruction of the long-axis plane of the left SCC (b) demonstrate a dehiscence of the bony covering of the SCC in the arcuate eminence (arrows). c Corresponding image example of a patient without SCC dehiscence with a clearly visible bony covering (dark hypointense line) of the SCC (arrows). SCC, superior semicircular canal.

general population [Masaki, 2011]. However, the majority of patients with SCD are asymptomatic. The prevalence of SCD syndrome with sound-induced vestibular symptoms, low-frequency conductive hearing loss, autophony, hyperacusis, and aural fullness amongst other symptoms is reported to be around $0.6 \%$ [Masaki, 2011].

Due to the relatively high prevalence of SCD, it seems likely that a certain number of patients receiving a cochlear implant (CI) would have it. Only one study has investigated the CI outcome in this specific patient population [Puram et al., 2015]. Puram et al. [2015] found a prevalence of 7\% of SCD among CI recipients and reported decreased postoperative word perception ability compared to CI recipients without SCD. Nonetheless, both groups had improved in word perception ability relative to their preoperative performance. The subjective rate of dizziness was comparable between both groups. The authors concluded that the surgical outcome between CI recipients with and without SCD is similar, although the average postoperative word perception ability was significantly lower in patients with SCD.

Computed tomography represents the gold standard for diagnosing SCD. However, multiple studies have demonstrated the efficacy of using magnetic resonance imaging (MRI) for its diagnosis [Browaeys et al., 2013; Inal et al., 2016; Beyazal Çeliker et al., 2018]. The aim of this study was to conduct a retrospective analysis of the prevalence of SCD among CI recipients, word perception outcome over time, and rate of preservation of residual hearing. We hypothesized that the CI outcome, hearing preservation, and word perception over time in patients with SCD would be the same compared to CI recipients without SCD. This is the first report of hearing preservation rates after cochlear implantation in patients with SCD.

\section{Methods}

The study was conducted in accordance with the Ethics Committee of Zurich (KEK-ZH-Nr. 2017-00852) and with international standards for human research. A retrospective chart review of 582 patients receiving cochlear implantations at the University Hospital of Zurich, Switzerland, between January 2003 and December 2013 was performed. Inclusion criteria were as follows: $\geq 18$ years, German speaking, residual hearing at any frequency before surgery, implantation with a full-length CI electrode array, preoperative 1.5- or 3-tesla MRI available including thin-sliced T2-weighted sequences of the temporal bone, postoperative assessment of residual acoustic hearing, and at least 3 postoperative monosyllabic word perception test scores after approximately 6,12 , and 18 or more months.

All cochlear implantations were carried out according to the standard surgical technique. Soft surgical principles were followed. The correct positioning of the electrode array was confirmed using a cochlear view X-ray. For the assessment of subjective dizziness after surgery, patients' charts were analyzed retrospectively.

\section{Audiometric Evaluation}

All audiological testing was conducted at the University Hospital of Zurich. Pure-tone testing was performed in concordance with ISO 8253-1. The pure-tone average (PTA) and hearing loss were calculated from the hearing thresholds at 250,500, and 1,000 $\mathrm{Hz}$. If no response was present, the maximum audiometer output $+5 \mathrm{~dB}$ was entered into the database. Functional residual hearing was defined as a PTA of $\leq 85 \mathrm{~dB}$ HL.

The postoperative word perception ability was assessed using the Swiss version of the German Freiburger monosyllabic test in quiet [Kompis et al., 2006]. The tests were conducted in the electric-only condition with the other ear masked. Percentage of words correctly repeated at a presentation level of $65 \mathrm{~dB}$ SPL was determined. If more than one word perception score was available after $\geq 18$ months, then the last assessment was chosen.

\section{Radiological Assessment}

Imaging was performed using 1.5- or 3-tesla MRI scanners from different vendors. The presence of SCD in the preoperative MRI was assessed by 2 independent investigators (J.M. and S.W.) 
Table 1. Comparison of demographic and audiometric data between patients without and with SCD

\begin{tabular}{llll}
\hline & Non-SCD & SCD & $p$ value \\
\hline Total, $n$ & 48 & 7 & $0.7^{\mathrm{a}}$ \\
Gender: female, $n(\%)$ & $27(56)$ & $6(85)$ & $0.7^{\mathrm{a}}$ \\
Side: right, $n(\%)$ & $25(52)$ & $3(43)$ & $0.0502^{\mathrm{b}}$ \\
Mean age at implantation (range), years & $50(19-80)$ & $63(31-89)$ & $0.35^{\mathrm{b}}$ \\
Mean duration of hearing loss (SD), years & $23(15)$ & $17(19)$ & $0.7^{\mathrm{a}}$ \\
Cochlear implant, $n$ & & 3 & $0.69^{\mathrm{a}}$ \\
$\quad$ CI24RE & 26 & 4 & $3(43)$ \\
\hline CI422 & 22 & $27(56)$ & \\
\hline
\end{tabular}

SCD, semicircular canal dehiscence. ${ }^{a}$ Fisher's exact test. ${ }^{b} t$ test with Bonferroni correction.

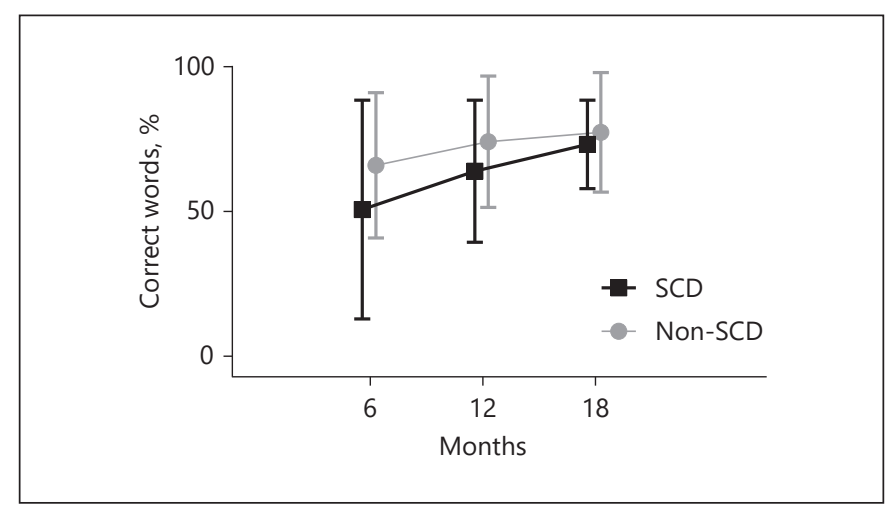

Fig. 2. Mean monosyllabic word perception score over time in the SCD and the non-SCD group. Percentage of words correctly repeated at a presentation level of $65 \mathrm{~dB}$ SPL is shown. SCD, semicircular canal dehiscence.

blinded to patient and outcome information. The SCD was evaluated with images in the parallel, perpendicular, and oblique sagittal planes to the semicircular canals [Beyazal Çeliker et al., 2018] (Fig. 1). The MRI findings were initially graded in 4 categories $(1=$ SCD , 2 = no SCD , 3 = suspicious for SCD, and $4=$ SCD unlikely). In patients with initial category 3 or 4 , both investigators revaluated the images independently and allocated it to either group 1 (non-SCD) or group 2 (SCD). After reevaluation, there was complete agreement between both investigators. Consequently, for the final analysis, all patients were allocated to either group 1 (non-SCD) or group 2 (SCD).

For this retrospective analysis, we had to rely on MRI findings for the diagnosis of SCD, as CT scans and vestibular evoked myogenic potential (VEMP) tests were not routinely performed before cochlear implantation at our institution. However, multiple studies comparing CT with MRI for the diagnosis of SCD showed a high reliability of MRI [Browaeys et al., 2013; Inal et al., 2016; Beyazal Çeliker et al., 2018]. Furthermore, MRI findings were matched with CT findings if available.

\section{Statistical Analysis}

Statistical analysis was conducted using GraphPad Prism Version 8.0.0 (GraphPad Software, Inc., San Diego, CA, USA). To compare demographic data between groups, either Fisher's exact test (gender, side, CI, and surgical approach) or a $t$ test with Bonferroni correction (mean age, mean duration of hearing loss, and mean preoperative PTA) was conducted. Postoperative outcome was analyzed using a $t$ test with Bonferroni correction (mean hearing loss and mean postoperative word perception ability).

\section{Results}

Fifty-five patients were included. Four hundred ninety-one patients had to be excluded due to incomplete audiological data, 5 patients had no MRI, and 31 patients had MRIs without thin-sliced T2-weighted images of the temporal bone. After initial analysis of the radiological data, 6 patients were in group 1, 43 in group 2,2 in group 3, and 4 in group 4 . After reevaluation, 7 patients (13\%) were allocated to group 1 (SCD group) and 48 patients $(87 \%)$ to group 2 (non-SCD group). MRI and CT scans were available in 14 cases (25\%). In all of these cases, there was agreement between MRI and CT results. All of these patients were allocated to group 2 (non-SCD group). Demographic data of both groups are summarized in Table 1. The demographic data showed no significant difference between group 1 and group 2.

Mean postoperative word perception scores were $66 \%$ in the non-SCD group versus $50 \%$ in the SCD group ( $p=0.17)$ after 6 months, 74 versus $64 \%(p=0.28)$ after 12 months, and 77 versus $73 \%(p=0.62)$ after 18 or more months (Fig. 2). Thirty patients (62\%) in the 


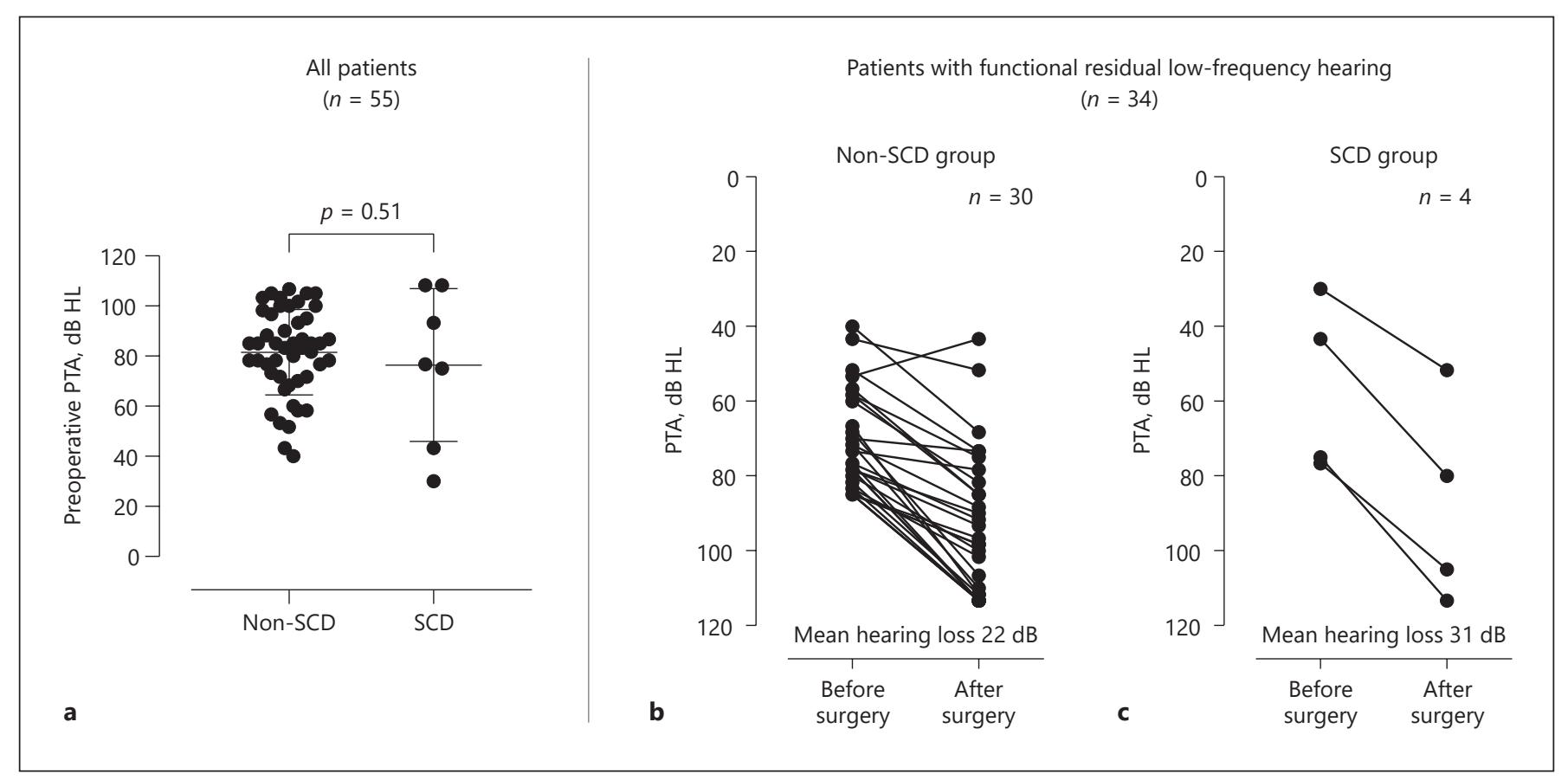

Fig. 3. Preoperative hearing in all patients (a) and pre- and postoperative hearing in the subgroup of patients with functional residual hearing before surgery $(n=34)(\mathbf{b}, \mathbf{c})$. SCD, semicircular canal dehiscence.

non-SCD group and 4 (57\%) in the SCD group had residual functional hearing. In these patients, the mean preoperative hearing was $81 \mathrm{~dB} H L(S D=17 \mathrm{~dB})$ in the non-SCD group and $79 \mathrm{~dB} H \mathrm{HL}(\mathrm{SD}=30 \mathrm{~dB})$ in the SCD group. Mean postoperative hearing loss 4 weeks after surgery was $22 \mathrm{~dB}(\mathrm{SD}=12.2 \mathrm{~dB})$ in the non-SCD group versus $31 \mathrm{~dB}(\mathrm{SD}=8 \mathrm{~dB})$ in the $S C D$ group $(p=0.15)$ (Fig. 3). Seven patients in the non-SCD group and 1 patient in the SCD group had a complete loss of residual hearing.

\section{Discussion}

The aim of this study was to determine the prevalence of asymptomatic SCD in CI recipients and describe the CI outcome in this population. Overall, there was no difference in postoperative word perception ability in the electric-only condition and rate of preservation of residual hearing between CI recipients without and with SCD. Our data suggest that asymptomatic SCD does not influence CI outcome. However, with a prevalence of $13 \%$, SCD seems to be more prevalent among CI recipients than among healthy adults (i.e., $3 \%$ as reported by Masaki [2011]).
Only one report about CI recipients with SCD has been published [Puram et al., 2015]. Compared to our results, Puram et al. [2015] reported a slightly lower prevalence of SCD among CI recipients (7\%) and found significantly lower $\mathrm{CNC}$ word repetition ability in patients with SCD versus those without SCD. However, the reference group and the SCD group in their study showed a significant difference regarding duration of hearing loss ( 249 vs. 494 months), which is a known factor influencing CI outcome [van den roek and Dunnebier, 2009]. Therefore, they accounted for the significant difference between both groups to be mainly due to a significantly longer duration of hearing loss in the SCD group. In our study population, duration of hearing loss was not significantly different between the SCD group and the non-SCD group, and we could not detect a significant difference in postoperative word perception ability at any time point.

Hearing preservation rate was unaffected by SCD. We analyzed hearing preservation in a subgroup of patients with functional residual hearing in the low frequencies (low-frequency PTA $\leq 85 \mathrm{~dB} \mathrm{HL}, n=34$ ) to minimize distortion of the results due to the ceiling effect. The postoperative hearing loss was comparable between both groups without a significant difference 
(mean hearing loss was $22 \mathrm{~dB}$ in the non-SCD group vs. $31 \mathrm{~dB}$ in the SCD group). It has to be noted that 1 patient in the non-SCD group showed an improvement of the hearing threshold of $10 \mathrm{~dB}$ after surgery (Fig. 3b). However, this patient suffered from fluctuating hearing loss due to Ménière's disease, which likely explains the postoperative improvement of the hearing threshold.

Our study has some limitations. First, it is a retrospectively conducted analysis and the number of patients is limited, especially in the SCD group. Further, we based our diagnosis of SCD solely on radiological findings. VEMP tests as a method to confirm the diagnosis of SCD were not available. Last, we relied on MRI rather than on CT results, although $\mathrm{CT}$ scans are the gold standard to diagnose SCD radiologically. The reason is that CT scans were also not routinely conducted before cochlear implantation at our institution and were therefore available only in $25 \%$ of our population. However, according to the available literature [Browaeys et al., 2013; Inal et al., 2016; Beyazal Çeliker et al., 2018], MRI represents a reliable tool to diagnose SCD. Furthermore, in the subgroup of $14 \mathrm{CI}$ recipients with MRI as well as CT scans, the assessment showed agreement between the results of both modalities. Overall - despite the mentioned limitations - this is the first study supporting the notion that $\mathrm{CI}$ outcome in asymptomatic SCD patients is equal to the CI outcome in non-SCD patients, especially with regard to word perception in the short term as well as the long term and to hearing preservation.

\section{Conclusions}

CI outcome is comparable between recipients with and without SCD. Specifically, hearing preservation rate and word perception ability in the electric-only condition seem not affected by SCD. The rate of progress of word perception ability in the first 12 months is not influenced by SCD.

\section{Statement of Ethics}

The study was conducted in accordance with the Ethics Committee of Zurich (KEK-ZH-Nr. 2017-00852) and with international standards for human research.

\section{Conflict of Interest Statement}

The authors disclose no conflicts of interest. There are no funding sources to declare.

\section{Author Contributions}

J.M. was responsible for study planning, radiological assessments, and writing the manuscript. S.W. was responsible for study planning, radiological assessments, and reviewing the manuscript. F.P. was responsible for study planning, statistical analyses, and reviewing the manuscript. C.R. and A.H. were responsible for performing CI surgeries and contributing to writing the manuscript. D.V. was responsible for study planning and reviewing the manuscript. A.D. was the initiator and leader of the study. He was also responsible for radiological assessments and writing the manuscript. All authors read and approved the final manuscript.

\section{References}

Beyazal Çeliker F, Özgür A, Çeliker M, Beyazal M, Turan A, Terzi S, et al. The efficacy of magnetic resonance imaging for the diagnosis of superior semicircular canal dehiscence. J Int Adv Otol. 2018;14(1):68-71.

Browaeys P, Larson TL, Wong ML, Patel U. Can MRI replace CT in evaluating semicircular canal dehiscence? Am J Neuroradiol. 2013; 34(7):1421-7.

Inal M, Burulday V, Bayar Muluk N, Kaya A, Şimşek G, Ünal Daphan B. Magnetic resonance imaging and computed tomography for diagnosing semicircular canal dehiscence. J Craniomaxillofac Surg. 2016;44(8):998-1002.
Kompis M, Krebs M, Häusler R. [Verification of normative values for the Swiss version of the Freiburg speech intelligibility test]. HNO. 2006;54(6):445-50.

Masaki Y. The prevalence of superior canal dehiscence syndrome as assessed by temporal bone computed tomography imaging. Acta Otolaryngol. 2011;131(3):258-62.
Minor LB, Solomon D, Zinreich JS, Zee DS. Sound- and/or pressure-induced vertigo due to bone dehiscence of the superior semicircular canal. Arch Otolaryngol Head Neck Surg. 1998;124(3):249-58.

Puram SV, Roberts DS, Niesten ME, Dilger AE, Lee DJ. Cochlear implant outcomes in patients with superior canal dehiscence. Cochlear Implants Int. 2015;16(4):213-21.

van den Broek E, Dunnebier EA. Cochlear implantation in postlingually hearing-impaired adults: choosing the most appropriate ear. Int J Audiol. 2009;48(9):618-24. 